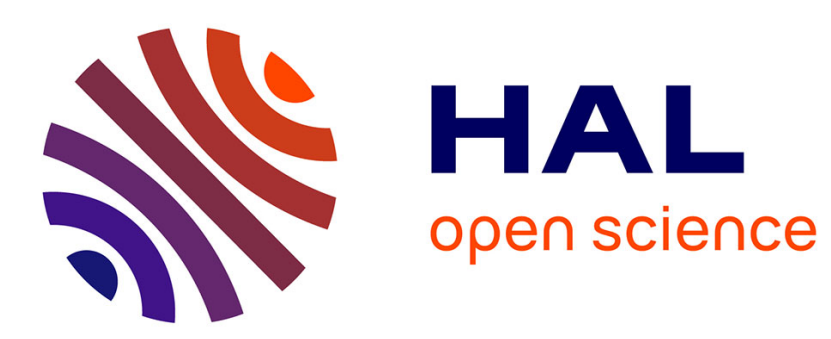

\title{
Predictor-based control of LTI remote systems with estimated time-varying delays
}

\author{
Yang Deng, Vincent Léchappé, Emmanuel Moulay, Franck Plestan
}

\section{To cite this version:}

Yang Deng, Vincent Léchappé, Emmanuel Moulay, Franck Plestan. Predictor-based control of LTI remote systems with estimated time-varying delays. IEEE Control Systems Letters, 2021, 5 (1), pp.289-294. 10.1109/LCSYS.2020.3001671 . hal-02871127

\section{HAL Id: hal-02871127 \\ https://hal.science/hal-02871127}

Submitted on 17 Jun 2020

HAL is a multi-disciplinary open access archive for the deposit and dissemination of scientific research documents, whether they are published or not. The documents may come from teaching and research institutions in France or abroad, or from public or private research centers.
L'archive ouverte pluridisciplinaire HAL, est destinée au dépôt et à la diffusion de documents scientifiques de niveau recherche, publiés ou non, émanant des établissements d'enseignement et de recherche français ou étrangers, des laboratoires publics ou privés. 


\title{
Predictor-based control of LTI remote systems with estimated time-varying delays
}

\author{
Yang Deng, Vincent Léchappé, Emmanuel Moulay, and Franck Plestan, Member, IEEE
}

\begin{abstract}
This paper provides a practical control solution to remote control systems with unknown time-varying delays. An external signal which can be considered as a specific communication loop is added to estimate the unknown round-trip delay in finite time. Then the system is stabilized with the practical delay estimation technique and a predictor-based controller. The main results of this paper also provide an alternative way to design predictor-based controller for systems with known input and output time-varying delays. The theoretical results of this paper are illustrated by simulation results.
\end{abstract}

Index Terms-Delay systems, Lyapunov methods, Estimation

\section{INTRODUCTION}

$\mathbf{C}$ ONTROL systems involving wireless communications, called "remote control systems (RCS)" [1, Chapter 3.3] are widely studied by the control community for the last decades (e.g. remote flight control [2], wireless power grid [3]). In such systems, the sensors and controllers are no longer physically connected to each others, they share information through telecommunications networks in order to reduce the costs. However, such communications often introduce latencies caused by protocols or communication channels. Thus, a RCS can be modeled as a time-delay system [4] with input and output delays.

In applications, the transmission delays are often time-varying, which makes the control design challenging. The work in $[5$, Chapter 6] considers the control of systems with a single input time-varying delay, and the authors of [6] study the output-feedback of systems with a single output time-varying delay, but these two methods can only cope with single slowvarying delay. For systems with both time-varying input and output delays, the results in [7] provide a predictor-based control solution and this method is extended in [8] in order to deal with the output-feedback case. However, [5], [6], [7], [8] cannot deal with unknown time-varying delays, the delays are supposed to be known or practically known (i.e. the known nominal delay values are sufficiently close to the real timedelays). In [9], the passification-based adaptive controller is adopted to stabilize a class of hyper-minimum-phase systems

Manuscript received Mar 17, 2020; revised May 18, 2020; accepted Jun 05, 2020. The work of Yang Deng was financed by China Scholarship Council (CSC).

Yang Deng and Franck Plestan are with Ecole Centrale de Nantes, LS2N UMR CNRS 6004, Nantes, France (e-mails: yang.deng@ec-nantes.fr, franck.plestan@ec-nantes.fr).

Vincent Léchappé is with Université de Lyon, INSA Lyon, Ecole Centrale de Lyon, Université Claude Bernard Lyon 1, CNRS, Ampère F-69621, Villeurbanne, France (e-mail: vincent.lechappe@insa-lyon.fr).

Emmanuel Moulay is with Université de Poitiers, XLIM UMR CNRS 7252, Poitiers, France (e-mail: emmanuel.moulay@univ-poitiers.fr). with unknown time-varying delays. This method is simple, but it also has two main drawbacks:

- it cannot stabilize system with arbitrarily long delays;

- it cannot deal with non-minimum-phase systems.

Although the control of RCS with unknown time-varying delays is challenging, some practical solutions can be made to simplify this problem. In [10], a specific communication loop is introduced to measure the round-trip delay of the system, the system clock is sent along with the control signal to the plant and along with the plant's output back to the controller, then one can measure the delay value by using the current clock and the received delayed clock. At the meantime, the delay measurement value is used in an adaptive Smith predictor [11] to stabilize the system. With this method, the unknown round-trip time-varying delay is technically measured thanks to the physical nature of the system. Inspired by the technique [10], another practical delay estimator [12] is introduced to estimate the round-trip delay, it is more robust than the delay measurement method in the presence of channel inherent noises [13], the detailed comparison between them are given in [12, Section IV-E]. The main objective of this work is to combine the practical delay estimation algorithm [12] and the predictor-based controller [5] to provide a practical control solution to the RCS with time-varying input and output delays. The theoretical results will show that the proposed practical control solution can stabilize systems with fast-varying output delays.

This paper is organized as follows, the frameworks of the control loop and the practical delay estimation loop are introduced in Section II, then the control problem is formulated in Section III in details. The main stability and convergence results are provided in Section IV, and the theoretical results are illustrated by simulation results given in Section V. Finally, a conclusion ends this paper with Section VI.

\section{FRAMEWORK OF THE CONTROLLER AND THE PRACTICAL DELAY ESTIMATOR}

In this section, the frameworks of the controller and the practical delay estimator are introduced, see Figure 1. The control solution presented in Figure 1 is comprised of two different loops:

- Control loop (black loop of Figure 1): This loop is the standard control loop of the system, the control signal $u(t)$ is transmitted to the plant through a channel that is subject to a time-varying delay $h_{i}(t)$. Thus, the delayed control signal $u\left(t-h_{i}(t)\right)$ is applied to the plant at the current instant, and then the state measurement $x(t)$ is 


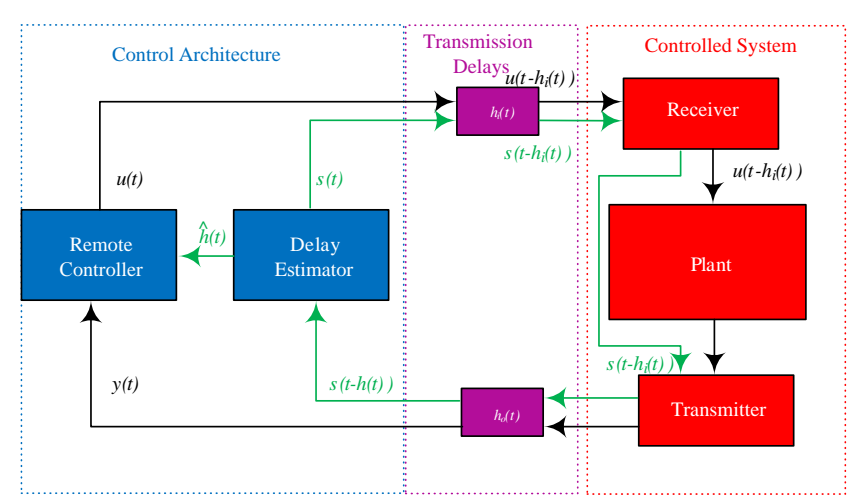

Fig. 1: Control and practical delay estimation of RCS with time-varying delays.

sent back to the controller. Due to the transmission delay $h_{o}(t)$, only the delayed state information $x\left(t-h_{o}(t)\right)$ is available to the controller.

- Delay estimation loop (green loop of Figure 1): This loop is a specific communication loop of the system, of which the only use is to estimate the unknown roundtrip delay, as done in [10], [12]. An external signal $s(t)$ is generated by the controller and transmitted to the plant along with the control signal $u(t)$. Therefore, the delayed signal $s\left(t-h_{i}(t)\right)$ is received by the plant of Figure 1. When the delayed control input $u\left(t-h_{i}(t)\right)$ is injected into the plant, the signal $s\left(t-h_{i}(t)\right)$ is transferred to the plant's transmitter, then the transmitter sends the state measurement $x(t)$ and the delayed signal $s\left(t-h_{i}(t)\right)$ back to the controller. Consequently, the delayed state $y(t)=x\left(t-h_{o}(t)\right)$ and the delayed signal $s(t-h(t))=$ $s\left(t-h_{o}(t)-h_{i}\left(t-h_{o}(t)\right)\right)$ are available to the controller at the same time, and $s(t-h(t))$ is used to compute the delay estimation $\hat{h}(t)$ which will be plugged into the control algorithm. In real applications, an additional DSP (Digital Signal Processor) is attached to the plant to build this specific communication loop, please see [10, Fig. 7] for details about the configuration.

The delay estimation loop has the following benefits:

- The delay estimation loop is isolated from the control loop, the external signal does not depend on the state or input information of the system. Moreover, the delay identifiability [14] of the system is no longer required. For instance, the work of [10] uses the system clock to measure the round-trip delay, the system clock $s(t)=t$ is independent of the system.

- It is not necessary to estimate $h_{i}(t)$ and $h_{o}(t)$ separately, only the round-trip delay is required by the controller. The synchronization between the controller and the plant is not required [10, Section III-A] since all of the computations are done on the controller.

\section{PROBLEM FORMULATION}

Throughout this paper, the following notations are used, the identity matrix with size $n \times n$ writes as $I_{n}$, and the Euclidean norm of a matrix is defined as $\|\cdot\|$. The maximum and minimum eigenvalues of a matrix $P \operatorname{read}$ as $\bar{\lambda}(P)$ and $\underline{\lambda}(P)$, respectively. According to [15], the notation $x_{t}(\theta)$ represents $x(t+\theta)$, with $\theta \in[-h, 0]$. The right-hand time-derivative of a function $f(\cdot)$ at instant $t-h(t)$ reads as $\dot{f}(t-h(t))$, and $\frac{d}{d t}(f(t-h(t)))$ denotes the right-hand time-derivative of the function composition $t \mapsto f(t-h(t))$. Recall the chain rule given in [16, Theorem 5.5], the relation between $\frac{d}{d t} f(t-h(t))$ and $\dot{f}(t-h(t))$ is given as

$$
\frac{d}{d t} f(t-h(t))=\dot{f}(t-h(t))(1-\dot{h}(t)) .
$$

The sign-function [17, eqs. (1.13)-(1.14)] satisfies

$$
\operatorname{sign}(x)=\left\{\begin{array}{l}
1, \quad \text { if } \quad x>0 \\
-1, \quad \text { if } \quad x<0
\end{array}\right.
$$

and $\operatorname{sign}(0) \in(-1,1)$. Consider the frameworks given in section II, the RCS is supposed to be linear time-invariant (LTI) and with full-state measurement:

$$
\left\{\begin{array}{l}
\dot{x}(t)=A x(t)+B u\left(t-h_{i}(t)\right), \\
y(t)=x\left(t-h_{o}(t)\right) .
\end{array}\right.
$$

The round-trip delay of the system (3) reads as

$$
h(t)=h_{o}(t)+h_{i}\left(t-h_{o}(t)\right) .
$$

System (3) is controlled by the predictor-based controller

$$
u(t)=K[\underbrace{e^{A \hat{h}(t)} x\left(t-h_{o}(t)\right)+\int_{t-\hat{h}(t)}^{t} e^{A(t-s)} B u(s) \mathrm{d} s}_{z(t)}]
$$

where $\hat{h}(t)$ is an estimation of the round-trip delay (4).

Remark 1. The predictor-based controller (5) has an integral term, which is an infinite-dimensional term that must be computed by numerical approaches. However, inappropriate numerical approach may lead to instability [18]. In order to overcome this issue, the trapezoid method given in [19, p.1548] is adopted in this paper.

The initial condition of the system (3) reads as

$$
x(\theta)=\phi_{x}(\theta), \quad u(\theta)=\phi_{u}(\theta), \quad \theta \in[-\bar{h}, 0)
$$

where $\bar{h}$ is the upper bound of the round-trip delay (4). In order to simplify the stability analysis, one chooses the initial condition of $z(t)$ such that

$$
\begin{cases}\left\|\phi_{u}(\theta)\right\| \leq\|K\|\left\|\phi_{z}(\theta)\right\|, & \theta \in[-\bar{h}, 0), \\ \left\|\dot{\phi}_{u}(\theta)\right\| \leq\|K\|\left\|\dot{\phi}_{z}(\theta)\right\|, & \theta \in[-\bar{h}, 0), \\ u(0)=K z(0) . & \end{cases}
$$

The super-twisting algorithm based delay estimator [12] is

$$
\dot{\hat{h}}(t)=1-\frac{1}{\dot{s}(t-\hat{h}(t))} w(t)
$$

where $w(t)$ satisfies that

$$
w(t)=-\lambda|\sigma(t)|^{0.5} \operatorname{sign}(\sigma(t))+w_{1}(t) .
$$


The term $\sigma(t)=s(t-\hat{h}(t))-s(t-h(t))$ is called the "sliding variable", and $w_{1}(t)$ given in (9) is determined by the following dynamics

$$
\dot{w}_{1}(t)=\left\{\begin{array}{l}
-w(t), \quad \text { if } \quad|w(t)|>\bar{W} \\
-\alpha \cdot \operatorname{sign}(\sigma(t)), \quad \text { if } \quad|w(t)| \leq \bar{W}
\end{array} .\right.
$$

According to [17, Theorem 4.5] and [12, Theorem 1], if $\lambda$, $\alpha, \bar{W}$ are sufficiently large, then after a finite time $t_{F}$, the convergences $\sigma(t)=\dot{\sigma}(t)=0$ and $\hat{h}(t)=h(t)$ are established. The following assumptions are necessary to the controller design, the delay estimation, and the stability analysis.

Assumption 1. The round-trip delay (4) is upper bounded (as stated after (6)) as well as its first and second derivatives. The derivatives of the time-delays are bounded with

$$
\left|\dot{h}_{i}(t)\right| \leq \delta_{i}, \quad\left|\dot{h}_{o}(t)\right| \leq \delta_{o} .
$$

Assumption 1 is not restrictive since the transmission delays of the real communication networks are usually bounded and slow-varying [20, Section 4].

Assumption 2. The external signal $s(t)$ is monotonic, it has bounded first and second derivatives, and its first derivative satisfies that

$$
\underline{\varepsilon} \leq|\dot{s}(t)| \leq \bar{\varepsilon}, \quad t \geq-\bar{h} .
$$

Since $s(t)$ is generated by the user, it is always possible to find an appropriate $s(t)$ satisfying (12) (e.g. if one sets $s(t)=$ $k t$, then one has $\underline{\varepsilon}=\bar{\varepsilon}=k$ ).

Assumption 3. The system (3) is stabilizable, and the matrix $K$ given in (5) makes $A+B K$ is Hurwitz. For all $c_{u}>0$, there always exists a symmetric positive definite matrix $P$, such that

$$
(A+B K)^{T} P+P(A+B K)=-c_{u} I_{n} .
$$

\section{MAin RESUlts}

The main results of this article are stated as follows.

Theorem 1. Consider the framework displayed in Figure 1, the RCS (3) is controlled by the control solution composed of the approximated predictor-based controller (5) and the practical delay estimator (8)-(9)-(10) based on the external signal $s(t)$. There exists a finite time $t_{F}>0$ such that the roundtrip delay (4) is perfectly estimated, and then the closed-loop system of $z(t)$ is globally uniformly exponentially stable

$$
\|z(t)\| \leq M_{1} \max _{s \in[-\bar{h}, 0]}\|z(s)\| e^{-\eta t}, \quad t \geq 0
$$

with positive constants $M_{1}, \eta$. Moreover, there also exists a positive constant $M_{2}$ such that the state of the system $x(t)$ globally converges to zero with decay rate $\eta$ :

$$
\|x(t)\| \leq M_{2} \max _{s \in[-\bar{h}, 0]}\|z(s)\| e^{-\eta t}, \quad t \geq 0
$$

Proof. The proof is divided into 3 parts: the first part studies the system behavior on the transient phase $t \in\left[0, t_{F}\right)$; the second part deals with the Lyapunov-Krasovskii analysis for all $t \geq t_{F}$; the third part derives the stability and convergence results (14)-(15) for all $t \geq 0$.
Part 1: Consider the derivative of $\sigma(t)$ and Assumption 1, then substitute (8) into the derivative gives that

$$
\begin{aligned}
\dot{\sigma}(t) & =\dot{s}(t-\hat{h}(t))(1-\dot{\hat{h}}(t))-\frac{d}{d t} s(t-h(t)) \\
& =-\frac{d}{d t} s(t-h(t))+w(t) .
\end{aligned}
$$

The proof begins with the bound on $\sigma(0)$ : according to Assumption 1 and the mean value theorem [16, Theorem 5.11], there exists a constant $r \in[\min \{\hat{h}(0), h(0)\}, \max \{\hat{h}(0), h(0)\}]$, such that

$$
\begin{aligned}
|\sigma(0)| & =|s(t-\hat{h}(0)-s(t-h(0)))|=|\dot{s}(t-r)||\hat{h}(0)-h(0)| \\
& \leq 2 \bar{\varepsilon} \bar{h}
\end{aligned}
$$

with the help of $|\dot{s}(t)| \leq \bar{\varepsilon},|h(0)| \leq \bar{h}$, and well-chosen initial condition $|\hat{h}(0)| \leq \bar{h}$. Therefore, $w(0)$ is bounded as follows:

$$
|w(0)| \leq \lambda \sqrt{2 \bar{\varepsilon} \bar{h}}+\left|w_{1}(0)\right|,
$$

then one sets $\bar{W} \geq \lambda \sqrt{2 \bar{\varepsilon} \bar{h}}+\left|w_{1}(0)\right| \geq|w(0)|$, the statements given in [17, Theorem 4.5] and [12, Theorem 1] ensure that:

- Delay estimation $\hat{h}(t)$ converges to the round-trip delay (4) in a finite time $t_{F}$ with sufficiently large $\lambda$ and $\alpha$.

- Since $|w(0)| \leq \bar{W}$, then the trajectories of $w(t)$ never leaves the segment $[-\bar{W}, \bar{W}]$ for all $t \geq 0$.

Then the delay estimation dynamic satisfies that

$$
|\dot{\hat{h}}(t)| \leq 1+\bar{W} / \underline{\varepsilon} \triangleq \bar{\delta}, \quad t \geq 0 .
$$

Differentiating $z(t)$ along the trajectories of the system (3) leads to

$$
\begin{aligned}
\dot{z}(t)= & (A+B K) z(t)+\left(\dot{\hat{h}}(t)-\dot{h}_{o}(t)\right) A z(t) \\
& -\left(\dot{\hat{h}}(t)-\dot{h}_{o}(t)\right) A \int_{t-\hat{h}(t)}^{t} e^{A(t-s)} B u(s) \mathrm{d} s \\
& +\left(1-\dot{h}_{o}(t)\right) e^{A \hat{h}(t)} B u(t-h(t)) \\
& -(1-\dot{\hat{h}}(t)) e^{A \hat{h}(t)} B u(t-\hat{h}(t)), \quad t \geq 0 .
\end{aligned}
$$

Consider the dynamic (20), the boundedness $\left|\dot{h}_{o}(t)\right| \leq \delta_{o}$, $|\hat{\hat{h}}(t)| \leq \bar{\delta}$, then as done in [21, Lemma A.1], study the scalar function

$$
N(t)=\left\{\begin{array}{l}
z^{T}(t) z(t), \quad t \geq 0 \\
\phi_{z}^{T}(\theta) \phi_{z}(\theta), \quad t \in[-\bar{h}, 0)
\end{array}\right.
$$

its dynamic along the trajectories of (20) satisfies that

$$
\begin{aligned}
\dot{N}(t)=2 & z^{T}(t) \dot{z}(t) \leq 2\|A+B K\|\|z(t)\|^{2}+2\left(\bar{\delta}+\delta_{o}\right)\|A\|\|z(t)\|^{2} \\
& +2\left(\bar{\delta}+\delta_{o}\right) e^{\|A\| \bar{h}}\|B K\|\|A\| \bar{h}\|z(t)\| \max _{s \in[t-\bar{h}, t]}\|z(s)\| \\
& +2\left(1+\delta_{o}\right) e^{\|A\| \bar{h}}\|B K\|\|z(t)\| \max _{s \in[t-\bar{h}, t]}\|z(s)\| \\
& +2(1+\bar{\delta}) e^{\|A\| \bar{h}}\|B K\|\|z(t)\| \max _{s \in[t-\bar{h}, t]}\|z(s)\| \\
\leq 2 & \left(\max _{s \in[t-\bar{h}, t]}\|z(s)\|\right)^{2}=2 b \max _{s \in[t-\bar{h}, t]} N(s), \quad t \geq 0
\end{aligned}
$$


with

$$
\begin{aligned}
b= & \|A+B K\|+\left(\delta_{o}+\bar{\delta}\right)\|A\| \\
& +e^{\|A\| \bar{h}}\|B K\|\left[\left(\delta_{o}+\bar{\delta}\right)\|A\| \bar{h}+\left(2+\delta_{o}+\bar{\delta}\right)\right] .
\end{aligned}
$$

The analysis given in [22, Appendix B, p.232-233] and [21, Lemma A1] ensure that the trajectories of $z(t)$ and $\dot{z}(t)$ satisfy

$$
\begin{aligned}
& \max _{s \in[t-\bar{h}, t]}\|z(s)\| \leq \max _{s \in[-\bar{h}, 0]}\|z(s)\| e^{b t}, \quad t \in\left[0, t_{F}\right), \\
& \max _{s \in[t-\bar{h}, t]}\|\dot{z}(s)\| \leq b \max _{s \in[-\bar{h}, 0]}\|z(s)\| e^{b t}, \quad t \in\left[0, t_{F}\right) .
\end{aligned}
$$

The proof of this part indicates that the trajectories of $z(t)$ and $\dot{z}(t)$ are bounded during the transient phase $t \in\left[0, t_{F}\right)$.

Part 2: Recall the previous work given in [12, Theorem 1], for all $t \geq t_{F}$, the practical delay estimator (8)-(9)-(10) establishes the 2-ideal sliding mode $\sigma(t) \equiv \dot{\sigma}(t) \equiv 0$. Then the following finite time delay estimation is obtained:

$$
\hat{h}(t) \equiv h(t), \quad \dot{\hat{h}}(t) \equiv \dot{h}(t), \quad t \geq t_{F} .
$$

By virtue of (25), the dynamic of $z(t)$ given in (20) now reads as

$$
\begin{aligned}
\dot{z}(t)= & (A+B K) z(t)+\delta(t) A z(t) \\
& +\delta(t) e^{A h(t)} B\left[u(t)-\int_{t-h(t)}^{t} \dot{u}(s) \mathrm{d} s\right] \\
& -\delta(t) A \int_{t-h(t)}^{t} e^{A(t-s)} B u(s) \mathrm{d} s, \quad t \geq t_{F}
\end{aligned}
$$

with $\delta(t) \triangleq \dot{h}(t)-\dot{h}_{o}(t)$. Consider the Lyapunov-Krasovskii functional

$$
V\left(z, u_{t}, \dot{u}_{t}, t\right)=V_{1}(z)+\beta V_{2}\left(u_{t}, t\right)+\gamma V_{3}\left(\dot{u}_{t}, t\right)
$$

with

$$
\begin{aligned}
V_{1}(z) & =z^{T}(t) P z(t), \\
V_{2}\left(u_{t}, t\right) & =\int_{t-\bar{h}}^{t}(s-t+\bar{h})\|u(s)\|^{2} \mathrm{~d} s, \\
V_{3}\left(\dot{u}_{t}, t\right) & =\int_{t-\bar{h}}^{t}(s-t+\bar{h})\|\dot{u}(s)\|^{2} \mathrm{~d} s .
\end{aligned}
$$

Differentiating $V_{1}(z)$ along the trajectories of (26), taking the norm of the derivative, and considering the fact $t-\bar{h} \leq t-h(t)$ yields to

$$
\begin{aligned}
\dot{V}_{1}(z) & \leq-\left(c_{u}-|\delta(t)| c_{1}-|\delta(t)| c_{2}\right)\|z(t)\|^{2} \\
& +\frac{|\delta(t)|}{2}\left[c_{3}\left(\|z(t)\|^{2}+\|v(t)\|^{2}\right)+c_{4}\left(\|z(t)\|^{2}+\|w(t)\|^{2}\right)\right]
\end{aligned}
$$

for all $t \geq t_{F}$, with $c_{1}=2\|P A\|, \quad c_{2}=2 e^{\|A\| \bar{h}}\|B K\|\|P\|$, $c_{3}=2\|A\| e^{\|A\| \bar{h}}\|B\|\|P\|, \quad c_{4}=2 e^{\|A\| \bar{h}}\|B\|\|P\|, \quad\|v(t)\|=$ $\int_{t-\bar{h}}^{t}\|u(s)\| \mathrm{d} s$, and $\|w(t)\|=\int_{t-\bar{h}}^{t}\|\dot{u}(s)\| \mathrm{d} s$. Next, consider the time-derivative of $V_{2}\left(u_{t}, t\right)$, by using the Jensen's inequality [23, Proposition B.8], it leads to

$$
\dot{V}_{2}\left(u_{t}, t\right) \leq \bar{h}\|K\|^{2}\|z(t)\|^{2}-\frac{1}{2 \bar{h}}\|v(t)\|^{2}-\frac{1}{2} \int_{t-\bar{h}}^{t}\|u(s)\|^{2} \mathrm{~d} s
$$

for all $t \geq t_{F}$. Similarly, the derivative of $V_{3}\left(\dot{u}_{t}, t\right)$ satisfies that $\dot{V}_{3}\left(\dot{u}_{t}, t\right) \leq \bar{h}\|\dot{u}(t)\|^{2}-\frac{1}{2 \bar{h}}\|w(t)\|^{2}-\frac{1}{2} \int_{t-\bar{h}}^{t}\|\dot{u}(s)\|^{2} \mathrm{~d} s, \quad t \geq t_{F}$.
For all $t \geq t_{F}$, taking the norm of (26), and squaring the right-hand side of the inequality, then using the power mean inequality [24, p.203, Theorem 1] gives

$$
\begin{aligned}
\|\dot{z}(t)\|^{2} \leq & c_{5}\|z(t)\|^{2}+|\delta(t)|^{2} c_{6}\|z(t)\|^{2} \\
& +|\delta(t)|^{2} c_{7}\|v(t)\|^{2}+|\delta(t)|^{2} c_{8}\|w(t)\|^{2}
\end{aligned}
$$

where $c_{5}=4\|A+B K\|^{2}, c_{6}=4\left(\|A\|+e^{\|A\| \bar{h}}\|B K\|\right)^{2}, c_{7}=$ $4\|A\|^{2} e^{2\|A\| \bar{h}}\|B\|^{2}$, and $c_{8}=4 e^{2\|A\| \bar{h}}\|B\|^{2}$. After computing the upper bounds of $\dot{V}_{1}(z), \dot{V}_{2}\left(u_{t}, t\right)$, and $\dot{V}_{3}\left(\dot{u}_{t}, t\right)$, the upper bound of the Lyapunov-Krasovskii functional (27) reads as

$$
\begin{gathered}
V\left(z, u_{t}, \dot{u}_{t}, t\right) \leq \bar{\lambda}(P)\|z(t)\|^{2}+\beta \bar{h} \int_{t-\bar{h}}^{t}\|u(s)\|^{2} \mathrm{~d} s \\
+\gamma \bar{h} \int_{t-\bar{h}}^{t}\|\dot{u}(s)\|^{2} \mathrm{~d} s .
\end{gathered}
$$

Combine (29), (30), (31), (32), and (33), the functional $V+$ $2 \eta V$ is upper bounded by

$$
\begin{aligned}
& \dot{V}+2 \eta V \leq-\left[c_{u}-|\delta(t)|\left(c_{1}+c_{2}+\frac{1}{2} c_{3}+\frac{1}{2} c_{4}\right)\right. \\
& \left.-\gamma \bar{h}\|K\|^{2}\left(c_{5}+c_{6}|\delta(t)|^{2}\right)-\beta \bar{h}\|K\|^{2}-2 \eta \bar{\lambda}(P)\right]\|z(t)\|^{2} \\
& -\left[\frac{\beta}{2 \bar{h}}-\frac{|\delta(t)|}{2} c_{3}-\gamma \bar{h}\|K\|^{2} c_{7}|\delta(t)|^{2}\right]\|v(t)\|^{2} \\
& -\left[\frac{\gamma}{2 \bar{h}}-\frac{|\delta(t)|}{2} c_{4}-\gamma \bar{h}\|K\|^{2} c_{8}|\delta(t)|^{2}\right]\|w(t)\|^{2} \\
& -\left[\frac{\beta}{2}-2 \beta \bar{h} \eta\right] \int_{t-\bar{h}}^{t}\|u(s)\|^{2} \mathrm{~d} s-\left[\frac{\gamma}{2}-2 \gamma \bar{h} \eta\right] \int_{t-\bar{h}}^{t}\|\dot{u}(s)\|^{2} \mathrm{~d} s
\end{aligned}
$$

for all $t \geq t_{F}$. Indeed, the inequality $\dot{V}+2 \eta V \leq 0$ holds when

- the parameters $\beta, \gamma$, and $\eta$ are sufficiently small;

- $|\delta(t)|$ is sufficiently small.

Remind that the parameters $\beta, \gamma$, and $\eta$ are the parameters of the Lyapunov-Krasovskii functionnals so they can be taken sufficiently small. By virtue of the chain rule $[16$, Theorem 5.5], the following boundedness is obtained:

$$
\begin{aligned}
|\delta(t)| \leq & \left|\frac{d}{d t} h_{i}\left(t-h_{o}(t)\right)\right|=\left|\left(1-\dot{h}_{o}(t)\right) \dot{h}_{i}\left(t-h_{o}(t)\right)\right| \\
& \leq \delta_{i}\left(1+\delta_{o}\right) .
\end{aligned}
$$

Thus, for all given bound $\delta_{o}$, there always exists a sufficiently small $\delta_{i}$, such that $|\delta(t)|$ is small enough to ensure that (34) is negative definite, which leads to the following convergence:

$$
V(t) \leq V\left(t_{F}\right) e^{-2 \eta\left(t-t_{F}\right)}, \quad t \geq t_{F} .
$$

Consequently, if the parameter $\delta_{i}$ is sufficiently small (i.e. $h_{i}(t)$ varies sufficiently slow), then the closed-loop system of $z(t)$ is exponential stable with decay rate $\eta$.

Part 3. Consider the bounds given in (24), it is possible to derive that

$$
\begin{gathered}
V\left(t_{F}\right) \leq \bar{\lambda}(P)\left\|z\left(t_{F}\right)\right\|^{2}+\beta \bar{h}^{2}\|K\|^{2}\left(\max _{s \in\left[t_{F}-\bar{h}, t_{F}\right]}\|z(s)\|\right)^{2} \\
\quad+\gamma \bar{h}^{2}\|K\|^{2}\left(\max _{s \in\left[t_{F}-\bar{h}, t_{F}\right]}\|\dot{z}(s)\|\right)^{2} \\
=\underbrace{\left[\bar{\lambda}(P)+\left(\beta+\gamma b^{2}\right) \bar{h}^{2}\|K\|^{2}\right] e^{2 b t_{F}}}_{M_{0}^{2}} \cdot\left(\max _{s \in[-\bar{h}, 0]}\|z(s)\|\right)^{2} .
\end{gathered}
$$


Combining (36) and (37) implies that

$$
\|z(t)\| \leq \underbrace{\frac{M_{0}}{\sqrt{\underline{\lambda(P)}}} e^{\eta t_{F}}}_{M_{1}} \max _{s \in[-\bar{h}, 0]}\|z(s)\| e^{-\eta t}
$$

for all $t \geq t_{F}$. During the transient phase $t \in\left[0, t_{F}\right)$, the term $e^{\eta\left(t_{F}-t\right)}$ is always larger than 1, then (24) implies that

$$
\|z(t)\| \leq \max _{s \in[-\bar{h}, 0]}\|z(s)\| e^{(b+\eta) t_{F}} e^{-\eta t}, \quad t \in\left[0, t_{F}\right) .
$$

Indeed, the right-hand side of (39) is smaller than $M_{1} \max _{s \in[-\bar{h}, 0]}\|z(s)\| e^{-\eta t}$ since

$$
M_{1} \geq \sqrt{\frac{\bar{\lambda}(P)}{\underline{\lambda}(P)}} e^{(b+\eta) t_{F}} \geq e^{(b+\eta) t_{F}} .
$$

Thus, inequality (38) hold s for all $t \geq 0$, and then the closedloop system of $z(t)$ is globally uniformly exponentially stable (GUES) with decay rate $\eta$ for all $t \geq 0$. Moreover, the stability of $z(t)$ implies the following inequality

$$
\max _{s \in[t-\bar{h}, t]}\|z(s)\| \leq M_{1} e^{\eta \bar{h}} \max _{s \in[-\bar{h}, 0]}\|z(s)\| e^{-\eta t}, \quad t \geq 0 .
$$

Now, move on to the state $x(t)$ of the original system, it is used to compute the predictor $z$ at a future instant $t+\tilde{h}$ with $\tilde{h} \in[0, \bar{h}]$. If $\tilde{h}>\bar{h}$, then $t+\tilde{h}-h_{o}(t+\tilde{h})>t$ that contradicts the expression of the predictor (5). Thus, the following equality is available

$$
x(t)=e^{-A h(t+\tilde{h})}\left[z(t+\tilde{h})-\int_{t+\tilde{h}-h(t+\tilde{h})}^{t+\tilde{h}} e^{A(t+\tilde{h}-s)} B u(s) \mathrm{d} s\right] .
$$

Taking the norm of (42), and applying (38), (41) gives that

$$
\begin{aligned}
\|x(t)\| & \leq e^{\|A\| \bar{h}}\left[\|z(t+\tilde{h})\|+e^{\|A\| \bar{h}}\|B K\| \bar{h} \max _{s \in[t+\tilde{h}-\bar{h}, t+\tilde{h}]}\|z(s)\|\right] \\
& \leq \underbrace{\left[e^{\|A\| \bar{h}}+e^{2\|A\| \bar{h}}\|B K\| \bar{h} e^{\eta \bar{h}}\right] M_{1}}_{M_{2}} \max _{s \in[\bar{h}, 0]}\|z(s)\| e^{-\eta t}
\end{aligned}
$$

for all $t \geq 0$. Thus, the closed-loop system of $x(t)$ converges exponentially to zero that ends the proof.

Theorem 1 ensures that the practical delay estimator [12] and the predictor-based controller (5) can stabilize RCS with unknown time-varying delays, even if the output delay is fastvarying (i.e. large $\delta_{o}$ is endured). The main results of Theorem 1 can also be extended to the predictor-feedback of systems with known time-varying delays.

Proposition 1. Consider the system (3) with known timevarying delays $h_{i}(t), h_{o}(t)$, and the round-trip delay $h(t)$ shown in (4). The predictor-based controller

$$
u(t)=K\left[e^{A h(t)} x\left(t-h_{o}(t)\right)+\int_{t-h(t)}^{t} e^{A(t-s)} B u(s) \mathrm{d} s\right]
$$

leads to the global uniform exponential stable closed-loop system, if the input-delay $h_{i}(t)$ varies sufficiently slowly.

Proposition 1 is similar to the main results of [7, Theorem 1], in which another version of the round-trip delay $h(t)=h_{i}(t)+h_{o}(t)$ is used to computed the controller (44). The stability conditions of them are similar, but the round-trip delay given in [7] is more difficult to measure or to estimate in application than the alternative one (4). Thus, Proposition 1 emphasizes another theoretical contribution of this work.

\section{Simulation RESUltS}

In this section, the double integrator system given in [7]:

$$
\left\{\begin{array}{l}
\dot{x}(t)=\left[\begin{array}{ll}
0 & 1 \\
0 & 0
\end{array}\right] x(t)+\left[\begin{array}{c}
0 \\
0.7
\end{array}\right] u\left(t-h_{i}(t)\right), \\
y(t)=x\left(t-h_{o}(t)\right)
\end{array}\right.
$$

with unknown time-varying delays

$$
h_{i}(t)=0.5+0.4 \sin (t), \quad h_{o}(t)=0.5+0.45 \sin (25 \cdot t)
$$

is considered. The three control schemes are tested:

- Without delay estimation: one does not estimate the time-varying delays, and uses the constant mean value of the round-trip delay $\hat{h}(t)=h_{m}=1.0$ s to build the control law (5);

- With delay estimator introduced in [25]: one uses the combination of the gradient descent based delay estimator $\dot{\hat{h}}(t)=\rho_{h}[u(t-\hat{h}(t))-u(t-h(t))] \dot{u}(t-\hat{h}(t))$ and controller (5) to stabilize the system.

- With proposed method: one uses the technique of Theorem 1 to estimate the unknown round-trip delay (with practical delay estimator (8)-(9)-(10)) and stabilize the system (with controller (5)).

The parameters of the simulations are chosen as:

- Parameters of the controller (5): the feedback matrix reads as $K=\left[\begin{array}{ll}-4.2857 & -5.7143\end{array}\right]$, and the initial conditions are defined by $\phi_{x}(\theta)=\phi_{z}(\theta)=\left[\begin{array}{ll}1 & 2\end{array}\right]^{T}$, and $\phi_{u}(\theta)=-1$ for all $\theta \in[-\bar{h}, 0)$;

- Parameters of the delay estimator given in [25]: the gain $\rho_{h}$ is set to $1.5 \times 10^{-4}$;

- Parameters of the delay estimator (8)-(9)-(10): the external signal is set to $s(t)=t$, the parameters read as $\lambda=180, \bar{W}=200, \alpha=20, \hat{h}(0)=0.5$, and $w_{1}(0)=0$.

The sampling period of the simulation is set to $T_{s}=0.001 \mathrm{~s}$, and the results are presented in Figure 2, it is observed that the control schemes of Theorem 1 and [25] have good control performances on the system, whereas the curve with control law (5) and constant $\hat{h}(t)=h_{m}=1.0$ s diverges. Thus, the proposed delay estimation technique is effective to the control of system (45) with time-varying delays (46). Moreover, Figure 2a shows that the practical solution given in Theorem 1 has better control performances (i.e. $x(t)$ converges exactly to the origin without oscillations) than the existing method [25]. Next, Figure $2 \mathrm{~b}$ claims that the round-trip delay is accurately estimated by the practical delay estimator (8)-(9)-(10), but the gradient descent method [25] has large estimation errors since the round-trip delay is fast-varying. The slight fluctuation of the estimation error (with the proposed delay estimator) is caused by the imperfect numerical implementation of the signfunction (2), please refer to [17], [12] for details.

Finally, although the output delay $h_{o}(t)$ and the round-trip 


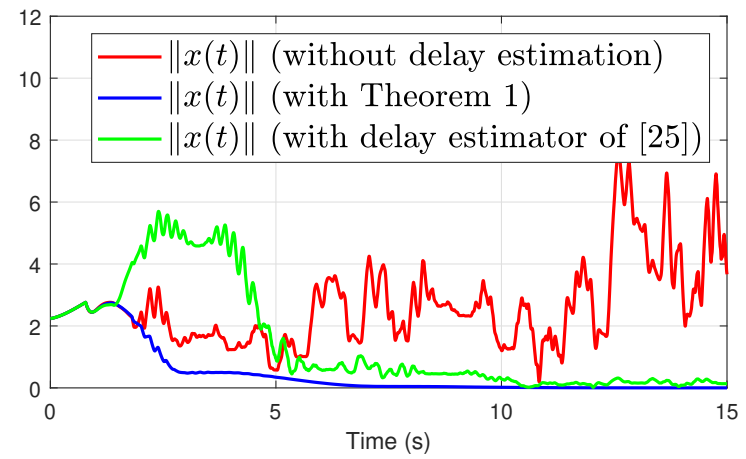

(a) State evolution of system (45) with three control schemes.

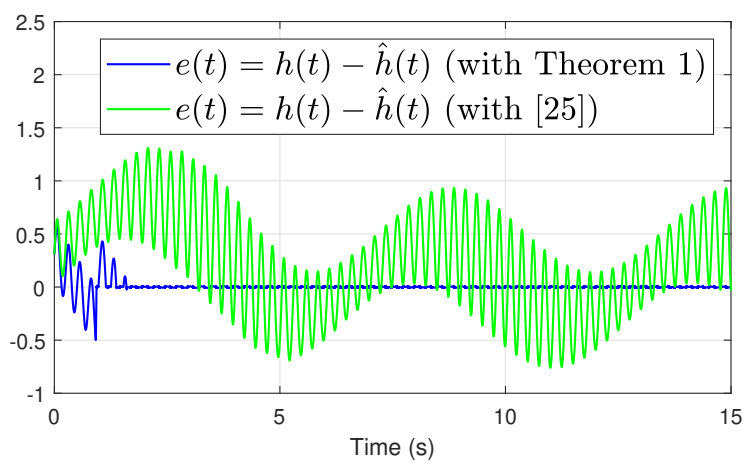

(b) Delay estimation error of the proposed method and [25].

Fig. 2: State evolution and delay estimation of different control solutions.

delay $h(t)$ are fast-varying (with derivative bound $\delta_{o}=11.25$ ), they cannot affect the closed-loop stability if the input-delay $h_{i}(t)$ is sufficiently slow-varying (with derivative bound $\delta_{o}=$ $0.4)$. Thus, the theoretical results provided by Proposition 1 are also illustrated by Figure 2 .

\section{CONCLUSION}

In this article, the practical delay estimation method given in [12] is combined with predictor-feedback to stabilize a class of remote control systems. The theoretical and numerical results illustrate that the round-trip delay estimated by [12] can be used to build control law for such systems. Moreover, Proposition 1 also provides an alternative choice of the predictor-based control with known time-varying input and output delays, which is more feasible in practice. The main results of this paper will be extended to the output feedback case (with partial state knowledge) for future works.

\section{REFERENCES}

[1] S.-H. Yang, Internet-based Control Systems: Design and Applications. Springer Science \& Business Media, 2011.

[2] D. W. Mellinger, Trajectory generation and control for quadrotors. Ph.D. Thesis of University of Pennsylvania, USA, 2012.

[3] A. M. Sarafi, G. I. Tsiropoulos, and P. G. Cottis, "Hybrid wirelessbroadband over power lines: A promising broadband solution in rural areas," IEEE Communications Magazine, vol. 47, no. 11, pp. 140-147, 2009.

[4] E. Fridman, Introduction to time-delay systems: Analysis and control. Springer, 2014.
[5] N. Bekiaris-Liberis and M. Krstic, Nonlinear control under nonconstant delays. Siam, 2013, vol. 25.

[6] R. Sanz, P. Garcia, and M. Krstic, "Observation and stabilization of ltv systems with time-varying measurement delay," Automatica, vol. 103 , pp. 573-579, 2019.

[7] V. Léchappé, E. Moulay, and F. Plestan, "Prediction-based control of LTI systems with input and output time-varying delays," Systems \& Control Letters, vol. 112, pp. 24-30, 2018.

[8] _ _ "Prediction-based control for LTI systems with uncertain timevarying delays and partial state knowledge," International Journal of Control, vol. 91, no. 6, pp. 1403-1414, 2018.

[9] A. Selivanov, E. Fridman, and A. Fradkov, "Passification-based adaptive control: Uncertain input and output delays," Automatica, vol. 54, pp. 107-113, 2015.

[10] C.-L. Lai and P.-L. Hsu, "Design the remote control system with the time-delay estimator and the adaptive smith predictor," IEEE Transactions on Industrial Informatics, vol. 6, no. 1, pp. 73-80, 2010.

[11] O. J. Smith, "A controller to overcome dead time," ISA Journal, vol. 6, pp. 28-33, 1959.

[12] Y. Deng, V. Léchappé, S. Rouquet, E. Moulay, and F. Plestan, "Super-twisting algorithm based time-varying delay estimation with external signal," IEEE Transactions on Industrial Electronics, 2019. [Online]. Available: 10.1109/TIE.2019.2960739

[13] C. E. Shannon, "Communication in the presence of noise," Proceedings of the IEEE, vol. 72, no. 9, pp. 1192-1201, 1984

[14] Y. Orlov, L. Belkoura, J.-P. Richard, and M. Dambrine, "On identifiability of linear time-delay systems," IEEE Transactions on automatic Control, vol. 47, no. 8, pp. 1319-1324, 2002.

[15] J. K. Hale and S. M. V. Lunel, Introduction to functional differential equations. Springer Science \& Business Media, 2013, vol. 99.

[16] T. M. Apostol, Mathematical analysis. Boston, USA: Addison-Wesley, 1974.

[17] Y. Shtessel, C. Edwards, L. Fridman, and A. Levant, Sliding mode control and observation. New York, USA: Springer, 2014.

[18] V. Van Assche, M. Dambrine, J.-F. Lafay, and J.-P. Richard, "Some problems arising in the implementation of distributed-delay control laws," in Proceedings of the 38th IEEE Conference on Decision and Control (Cat. No. 99CH36304), vol. 5. IEEE, 1999, pp. 4668-4672.

[19] D. Bresch-Pietri, J. Chauvin, and N. Petit, "Adaptive control scheme for uncertain time-delay systems," Automatica, vol. 48, no. 8, pp. 15361552, 2012.

[20] G. Fiengo, D. G. Lui, A. Petrillo, and S. Santini, "Distributed leadertracking adaptive control for high-order nonlinear lipschitz multi-agent systems with multiple time-varying communication delays," International Journal of Control, pp. 1-13, 2019.

[21] Y. Deng, V. Léchappé, E. Moulay, and F. Plestan, "State feedback control and delay estimation for LTI system with unknown input-delay," International Journal of Control, no. just-accepted, pp. 1-18, 2019. [Online]. Available: https://doi.org/10.1080/00207179.2019.1707288

[22] D. Bresch-Pietri, F. Mazenc, and N. Petit, "Robust compensation of a chattering time-varying input delay with jumps," Automatica, vol. 92, pp. 225-234, 2018.

[23] K. Gu, J. Chen, and V. L. Kharitonov, Stability of time-delay systems. Birkhäuser, 2003.

[24] P. S. Bullen, Handbook of means and their inequalities. Springer Science \& Business Media, 2013, vol. 560.

[25] V. Léchappé, S. Rouquet, A. Gonzalez, F. Plestan, J. De León, E. Moulay, and A. Glumineau, "Delay estimation and predictive control of uncertain systems with input delay: Application to a DC motor," IEEE Transactions on Industrial Electronics, vol. 63, no. 9, pp. 5849-5857, 2016. 